\title{
Ethik und Psyche: Die theoretischen Grundlagen des Umgangs mit geistigen Aspekten des Menschseins
}

\author{
Thomas Schramme
}

Online publiziert: 30. Juni 2012

(C) Springer-Verlag 2012

Von der Psyche reden heute nicht mehr viele Menschen; es scheint ein antiquierter, ja irgendwie peinlicher Begriff. So sprechen wir stattdessen lieber über das Gehirn und Neuronen, wenn wir uns auf geistige Eigenschaften des Menschen beziehen, denn wir haben gelernt, dass wir keine Dualisten sein dürfen. Kein Dualist zu sein heißt heutzutage, nur über den Körper und dabei natürlich insbesondere das Gehirn zu sprechen. Die mentale Begrifflichkeit, mit der wir gewohnt sind zu operieren, etwa wenn wir über Wünsche oder Überzeugungen sprechen, wird allenfalls noch als sogenannte Alltagspsychologie akzeptiert, die vermeintlich im Gegensatz zur wissenschaftlichen Psychologie steht. Kein Dualist zu sein heißt demnach, die Psyche eigentlich zu vergessen. Die Verwirrung und Verunsicherung, die hier herrscht, beruht letztlich auf einer theoretischen bzw. philosophischen Verwirrung. Was heißt es, kein Dualist zu sein? Du sollst nicht zwischen Körper und Geist unterscheiden - so wird das Credo gelesen. Descartes hat es falsch gemacht - darüber sind wir uns einig. Doch in welcher Weise soll nicht mehr zwischen beiden unterschieden werden? Descartes hatte verschiedene Existenzweisen des Körpers und der Seele unterstellt. Diese Sichtweise ist zu Recht überholt. Doch können wir tatsächlich alle Eigenschaften des Mentalen, wie etwa das subjektive Erleben von Schmerz, auf körperlicher Ebene erklären? Darüber gibt es in der Philosophie eine ausgedehnte Diskussion. Kaum einer der Beteiligten würde die Verabschiedung des Cartesianischen Dualismus zum Anlass nehmen, nur noch auf das Gehirn zu starren. Der sogenannte nicht-reduktive Physikalismus ist die vorherrschende Position in der philosophischen Debatte; doch für solche Komplexitäten besteht in Medizin und Feuilleton kein Bedarf. Das wäre nicht allzu schlimm, wenn der Streit um die Relation von Körper und Geist nur die Philosophie anginge. Doch jede Position in dieser Debatte ist in Bezug auf die menschliche Praxis nicht unschuldig. Wie wir die Psyche jeweils konzeptualisieren, hat Auswirkungen darauf, wie wir mit ihr interagieren.

Die genannte Verunsicherung bezüglich der vermeintlich richtigen begrifflichen Fassung der geistigen Aspekte des Menschseins hat die medizinische Teildisziplin, die sich damit

Prof. Dr.T. Schramme $(\bowtie)$

Philosophisches Seminar, Universität Hamburg, Von-Melle-Park 6, 20146 Hamburg, Deutschland

E-Mail: thomas.schramme@uni-hamburg.de 
befasst, also die Psychiatrie, lange geprägt. Die insbesondere in den Siebziger Jahren des Zwanzigsten Jahrhunderts geführte Auseinandersetzung um die Wissenschaftlichkeit des grundlegenden Konzepts der Psychiatrie, nämlich des Begriffs der psychischen Krankheit, ist zwar zugunsten einer weitgehenden Somatisierung der Psychopathologie inzwischen ad acta gelegt worden, hat aber insofern seine Spuren hinterlassen, als man sich nun vorsichtiger ausdrückt, und statt von Krankheiten offiziell lieber von Störungen spricht.

Möglicherweise hat diese Verunsicherung auch dazu geführt, dass die Psychiatrie nach wie vor ein vergleichsweise eher wenig beackertes Gebiet der Medizinethik ist. Dabei zeigt sich gerade an diesem Gegenstand eine analytisch und argumentativ hochwertige Auseinandersetzung als dringend erforderlich. Schließlich beruhen einige der dort zu verhandelnden ethischen Fragestellungen auf grundlegenden theoretischen Weichenstellungen und Problemen. Ganz allgemein gesprochen führt die Tatsache, dass uns der menschliche Geist nicht direkt zugänglich ist, zu einer weitgehend offenen Möglichkeit der Konzeptualisierung mit entsprechenden Auswirkungen. Der körperliche Aufbau des menschlichen Organismus lässt dem gegenüber weit weniger Interpretationsspielraum.

Beispielsweise wurde die Rationalität spätestens seit der Aufklärung zu einem, wenn nicht dem entscheidenden Merkmal der menschlichen Selbstbestimmung. Die Einschätzung der Einwilligungskompetenz von psychisch kranken oder auch dementen Patienten beruht entsprechend auf einer bestimmten Auffassung bezüglich der dafür notwendigen kognitiven Fähigkeiten. Das führte dazu, dass psychisch kranke Patienten zum Teil per definitionem aus der Gruppe der Einwilligungsfähigen ausgeschlossen wurden. Auch wenn inzwischen diese Extreme abgeschafft sind, nachdem einige Autoren immer wieder auf die Graduierbarkeit und Kontextspezifität des Kompetenzbegriffs verwiesen, so bleibt doch eine bestimmte Deutung vorherrschend, die unter anderen historischen und kulturellen Vorprägungen auch hätte anders aussehen können.

Mir geht es bei diesem Hinweis nicht darum zu bezweifeln, dass es gute Gründe für eine kognitive Deutung der Einwilligungskompetenz gibt, sondern ich möchte illustrieren, inwiefern die Konzeptualisierung der Psyche sich unserem Denken und Handeln verdankt. Entsprechend beruhen die ethischen Fragen, welche die Psyche betreffen, also etwa das Thema der Zwangsbehandlung in der Psychiatrie, aber auch des Neuro-Enhancements, auf spezifischen theoretischen Annahmen, die es zu diskutieren und zu problematisieren gilt. Dies geschieht aber allzu selten.

Ähnliche Überlegungen kann man in Bezug auf das neuerdings wieder in die Diskussion geratene Hirntodkriterium anführen. Auch hier zeigt sich, dass vermeintlich rein wissenschaftliche Herangehensweisen letztlich auf menschlichen Sichtweisen und Werten beruhen. Hiermit ist keineswegs die Frage der Entnahme von Organen gemeint, an der viele Menschen natürlich ein Interesse haben, sondern wesentlich grundlegender die Tatsache, dass die Identität von Geist und Gehirn, die wir heutzutage sicherlich mit guten Gründen postulieren, gleichwohl nur eine Sichtweise in der Debatte um das Leib-Seele Problem darstellt. Diese Sichtweise muss sich mit Gründen beweisen und sich an den Meinungen der Bevölkerung bewähren. Insofern es keine philosophische Lösung des Problems gibt, kann es auch keine wissenschaftspolitische geben. Das vorherrschende identitätstheoretische Paradigma sollte also nicht einfach akzeptiert, sondern kritisch diskutiert werden. Die ethische Debatte über das Hirntodkriterium verlangt dabei eine wesentlich tiefer gehende theoretische Fundierung, als es üblicherweise der Fall ist (vgl. aber [2]).

Darüber hinaus stellt sich die Frage, ob die medizinethischen Instrumente und Methoden, wie wir sie stellvertretend im Vier-Prinzipien-Ansatz von Beauchamp und Childress vor Augen haben, überhaupt ohne Abstriche auf den Bereich der Psychiatrie und allgemein der 
Psyche übertragen werden können. Existieren nicht dort spezifische Charakteristika, die eine genuine Ethik der Psyche verlangen?

Nehmen wir wieder ein Beispiel aus der Psychiatrie. Wir haben es dort häufig mit Patienten zu tun, die in besonderer Weise vulnerabel sind, da sie beispielsweise ambivalent in ihren Wünschen oder unsicher bezüglich ihrer Identität sind. Ja, es existiert sogar eine Extremform der Persönlichkeitsstörung, die Dissoziative Identitätsstörung, in der anscheinend mehrere Selbst in einem Körper hausen. Unsere üblichen medizinethischen Theorien kennen aber meist nur ein schwarz-weißes Vorgehen, das solche Grauzonen vernachlässigt. Ein Patient ist einwilligungsfähig oder nicht, er hat einen Wunsch oder nicht, er hat die oder jene identitätsstiftenden Wertvorstellungen oder nicht; für ambivalente und unsichere Aspekte des Personseins existiert da wenig Spielraum. So bleibt uns etwa in der medizinethischen Debatte über die Zulässigkeit des Suizids nicht viel mehr übrig, als die Frage nach der Entscheidungsfähigkeit und der rationalen Nachvollziehbarkeit des Todeswunsches zum nahezu allein entscheidenden Gesichtspunkt zu erklären. Doch sind Menschen, die einen Suizid in Erwägung ziehen, selten bestimmt in ihrer Entscheidung (vgl. [1]).

In ähnlicher Weise sind wir es gewohnt, Verbesserungen von psychischen Fähigkeiten wie Gedächtnis, Konzentrationsfähigkeit oder auch Einfühlungsvermögen in Analogie zu Fragen des körperlichen Enhancements zu diskutieren. Doch die geistigen Aspekte des Menschseins sind - zumindest teilweise - in ganz anderer Weise mit unserer Identität und unserem Selbstverständnis verknüpft, als es körperliche Eigenschaften sind. Sollte man beispielsweise in die Lage versetzt werden, sich an sehr viel mehr Aspekte seines Lebens zu erinnern, so wird man tatsächlich eine andere Person sein, in dem Sinne, dass man damit eine andere Lebensgeschichte erhält. Die ethische Frage, ob man eine solche identitätsverändernde Intervention durchführen können soll, ist mit den dürren Begriffen des Schadensverbots und ähnlichen Konzepten nur schwer einzuholen.

Was wir also benötigen ist eine Reflexion auf die theoretischen Grundlagen unseres Verständnisses der Psyche sowie der damit einhergehenden Färbungen der ethischen Debatte. Darüber hinaus sollte die Wirkkraft unserer medizinethischen Theorien für den Bereich der Psychiatrie und der ethischen Fragen in Bezug auf die Psyche insgesamt kritisch geprüft werden. Ob wir dann gleich eine eigenständige Ethik der Psyche für notwendig erachten werden, sei einmal dahingestellt.

Die diesjährige Jahrestagung der Akademie für Ethik in der Medizin in Hamburg stellt sich daher dem Thema Ethik und Psyche. Sie wird hoffentlich einen weiteren, wenn auch natürlich keineswegs den ersten Schritt hin zu einer stärkeren Fokussierung auf die theoretischen und ethischen Besonderheiten des menschlichen Umgangs mit der Psyche darstellen.

\section{Literatur}

1. Schlimme J (Hrsg) (2007) Unentschiedenheit und Selbsttötung: Vergewisserungen der Suizidalität. Vandenhoeck \& Ruprecht, Göttingen

2. Stoecker R (2010) Der Hirntod: Ein medizinethisches Problem und seine moralphilosophische Transformation, Studienausgabe. Alber, München 